\title{
A Versatile All-in-One Automated Processor for Electron Microscopy
}

\author{
Thomas E Strader ${ }^{1}$, Nicholas R Stewart ${ }^{1,2}$, Benjamin K August ${ }^{1}$, Steven L Goodman ${ }^{1}$ \\ 1. Microscopy Innovations, LLC, Marshfield, WI USA \\ 2. University of Wisconsin-Madison School of Medicine and Public Health, Madison, WI USA
}

Sample preparation for biological TEM is a long and tedious process requiring 1-2 days of hands-on time and effort, or partially accomplished with automated tissue processors that still require substantial manual intervention. Microwave oven processing accelerates reaction times and thus reduces overall preparation time to several hours, but still requires repeated manual reagent exchanges and considerable specimen handling. Further, these instruments and methods have limited capability for simultaneously preparing multiple specimens with different protocols, preparing both specimens and TEM grids, and preparing tissue specimens for serial block face Scanning Electron Microscopy.

The affordable benchtop mPrep ASP-1000 Automated Specimen Processor was developed to provide fully automated specimen and grid preparation for large and small research and clinical labs. The ASP was designed to perform almost any specimen and grid protocol, and to achieve rapid high-throughput sample preparation with the speed of microwave methods while providing "walk away" automation. Specimens in labeled mPrep/s specimen capsules (or TEM grids in mPrep/g capsules) are attached to the 8-capsule ASP robotic arm (Figure 1). Then, under computer control, the mPrep capsules are moved sequentially to reagents arranged in 96-well plates. Following reagent processing from fixation through resin infiltration, specimens in $\mathrm{mPrep} / \mathrm{s}$ capsules are then transferred to an oven for final polymerization.

Rapid specimen preparation is achieved with hundreds of repeated reagent exchanges to drive diffusion while consuming minimal reagent. This provides high quality reproducible preparation from fixation through epoxy infiltration. Mammalian muscle (Figure 2), yeast pellets in agar (Figure 3), and many other tissues (not shown) are reproducibly prepared in about 2 hours, while kidney can be processed from fixative to epoxy infiltration in just 45 minutes (Figure 4). Tissues can also be prepared for immuno-labeling by using LR White acrylic resin in only 45 minutes, as shown with muscle (Figure 5) and nervous tissue (Figure 6). These preparation times are comparable to the speed of microwave processing but without requiring the frequent manual reagent exchanges of lab microwave oven processing, and without the need to transfer resin infiltrated specimens into embedding molds prior to thermal or microwave polymerization since specimens remain in $\mathrm{mPrep} / \mathrm{s}$ capsules.

The ASP enables eight specimens (or grids) to be processed in parallel with each being treated with different reagents in a single processing run. This has been used to optimize specimen preparation protocols [1], and to simultaneously prepare titrations and controls for immuno-labeling [2]. The ASP has also recently been used to develop rapid protocols for en bloc SEM tissue preparation that reduce preparation time from 7 to 1.5 days [3].

The ASP can meet a wide range of processing needs in large and small labs. It can process tissues and ager pelleted cells very quickly and without manual reagent exchanges or tedious transfers of resininfiltrated specimens into embedding molds. It can also perform complex protocols with automated precision. Some applications not shown include conventional grid staining, en block immunolabeling, negative staining, and the uniform application of gold fiducials for 3D tomography [2]. 

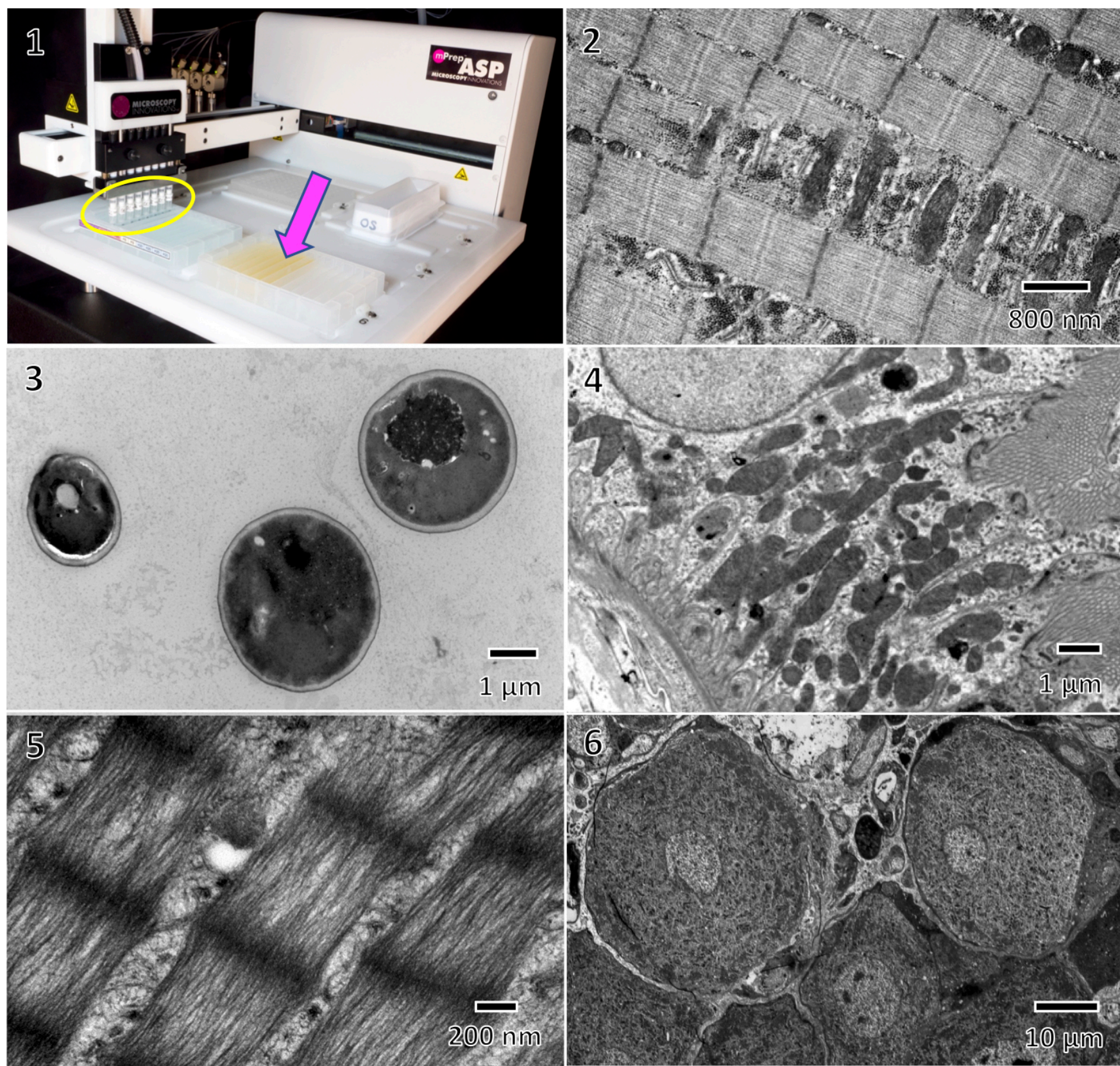

Figure 1. Tissue specimens in mPrep capsules on ASP-1000 robotic arm (circled). Reagents are drawn from the microwell plates (example arrow) arranged on ASP deck.

Figure 2. Rat gastrocnemius (skeletal) myofibril embedded in epoxy. Note mitochondria, Z \& M-lines.

Figure 3. Yeast cells in agar pellet embedded in epoxy.

Figure 4. Rat kidney embedded in epoxy with invaginating membrane folds \& elongated mitochondria.

Figure 5. Mouse sarcomeres from extraocular muscle embedded in LR White resin (without $\mathrm{OsO}_{4}$ ).

Figure 6. Mouse neuronal somas from lumbar dorsal root ganglion in LR White resin (without $\mathrm{OsO}_{4}$ ).

\section{References:}

[1] M McClain (2014). Microsc. Microanal. 20 (2014) 1288-9.

[2] EB Frankel, BK August, A Audhya, TE Strader Microsc. Microanal, 22 (2016) 1010-1.

[3] M McClain, SH Nowotarski, et al. Microsc. Microanal. 23 (2017) Post deadline poster 59. 